\title{
Back to the future: the absolute quantification of cerebral metabolic rate of glucose
}

\author{
Valentina Berti · Eleonora Vanzi · Cristina Polito • \\ Alberto Pupi
}

Received: 8 May 2013/Accepted: 15 July 2013/Published online: 30 July 2013

(C) Italian Association of Nuclear Medicine and Molecular Imaging 2013

\begin{abstract}
Quantitative maps of the cerebral metabolic rate of glucose (CMRGlc) derived from 2-18F-fluoro-2deoxy-D-glucose $\left({ }^{18} \mathrm{~F}-\mathrm{FDG}\right)$ positron emission tomography (PET) images are useful in brain studies, but practical methods are needed to allow their use in clinical routine. This review looks at the kinetic model at the basis of absolute quantification of CMRGlc and at the main methods for measuring CMRGlc using ${ }^{18}$ F-FDG PET, also focusing on some critical steps in the procedure.
\end{abstract}

Keywords CMRGlc Absolute quantification ·

Sokoloff · Patlak

\section{Introduction}

Ever since its introduction, positron emission tomography (PET) with 2-18F-fluoro-2-deoxy-D-glucose $\left({ }^{18} \mathrm{~F}-\mathrm{FDG}\right)$ has shown itself to be one of the neuroimaging techniques best suited to non-invasive investigation of brain function. A very important aspect of PET imaging is that it is quantitative: it has the capability of providing high-resolution images and allows the absolute quantification of local concentrations of tracer radioactivity in the brain.

There is a body of literature data demonstrating that ${ }^{18} \mathrm{~F}$ FDG PET brings added value to the diagnostic evaluation of neurodegenerative disorders over and above a routine clinical assessment, having sensitivity and specificity values of more than $85 \%[1,2]$. Over the past decade, the use

V. Berti $(\square) \cdot$ E. Vanzi · C. Polito · A. Pupi

Nuclear Medicine Unit, Department of Biomedical,

Experimental and Clinical Sciences, University of Florence,

Viale Morgagni 50, 50134 Florence, Italy

e-mail: valentina.berti@unifi.it of ${ }^{18}$ F-FDG PET in the evaluation of patients with dementia has improved diagnostic accuracy of these conditions, increased the possibilities of differential diagnosis, and led to earlier treatment, better therapy planning, and less uncertainty in diagnosis [3].

Many brain ${ }^{18}$ F-FDG PET studies are based on the qualitative evaluation of regional tracer uptake. However, while visual assessment continues to play a pivotal role, other PET image analysis methods could be applied to improve the diagnostic accuracy of ${ }^{18}$ F-FDG PET. Voxel-based analyses and semi-quantitative measurements of cerebral ${ }^{18} \mathrm{~F}$-FDG uptake, for example, have been successfully used both in research and in day-to-day clinical practice, and are especially useful for the identification of specific patterns of hypometabolism in different neurodegenerative disorders [1]. In order to produce semi-quantitative measures, these approaches usually employ a normalization procedure, i.e., a rescaling to the activity value either of the global mean of all gray matter voxels, or of a reference region (cerebellum or white matter). However, in patients with significant global reductions of brain metabolic activity, such as patients with neurodegenerative dementia, this procedure is liable to generate several errors; for example, the impression of metabolic increases in regions in which metabolism is actually unchanged.

Absolute quantification of brain glucose utilization is a more complex procedure that is able to provide fully quantitative estimates of the cerebral metabolic rate of glucose (CMRGlc). Fully quantitative measures provide invaluable information not only in clinical practice but also in clinical trials, and they are essential whenever a rigorous and objective parameter is desirable in order to accurately monitor disease progression [4] or assess potentially protective effects of treatments $[5,6]$.

The initial dynamic quantitative approaches with ${ }^{18} \mathrm{~F}$ FDG PET intended for calculating the absolute CMRGlc 
were already reliable; however, they were technically challenging and time-consuming, involving arterial blood sampling at multiple time points and complex mathematical procedures. Since then, ${ }^{18}$ F-FDG PET quantitative imaging has been constantly improved to refine PET acquisition and sampling procedures, making these simpler and feasible even in clinical settings, yet reasonably accurate.

This review looks at the kinetic model at the basis of the absolute quantification of CMRGlc and at the main methods for measuring CMRGlc using ${ }^{18} \mathrm{~F}$-FDG PET, also focusing on some critical steps in the procedure.

\section{Kinetic model}

Even though there is a clear correlation between ${ }^{18} \mathrm{~F}-\mathrm{FDG}$ PET signal and glucose consumption in brain tissue, the measured concentration of tracer uptake in this tissue is not a direct measurement of this biological function. In order to extract the biological measurement of interest, the PET data need to be evaluated using a mathematical model of ${ }^{18} \mathrm{~F}$ FDG behavior. This model needs to take into account both the delivery of the tracer to the tissue (which constitutes the input function) and its subsequent fate in brain tissue.

The compartmental model used for the quantitative estimation of CMRGlc using ${ }^{18} \mathrm{~F}$-FDG is an adaptation of the original model developed by Sokoloff during his pioneering work on glucose analogs 14C-deoxyglucose and ${ }^{18}$ F-FDG [7, 8]. Application of this kinetic model at the measurables of the system (input function and brain activity curve) generates CMRGlc estimates and also the individual kinetic constants, and thereby provides valuable insight into the various components of glucose metabolism, such as transport and phosphorylation. The tracer kinetic modeling of ${ }^{18} \mathrm{~F}-\mathrm{FDG}$ is based upon a standard threecompartment kinetic model, which can be reduced to the configuration shown in Fig. 1.

The first compartment $C_{\mathrm{a}}$ represents the arterial activity concentration of free ${ }^{18} \mathrm{~F}-\mathrm{FDG}$ in plasma; this is an open compartment that cannot be predicted within the model, and therefore the input function (IF) must be measured separately. The other two compartments are in the tissue, and for this reason the term "two tissue compartment"

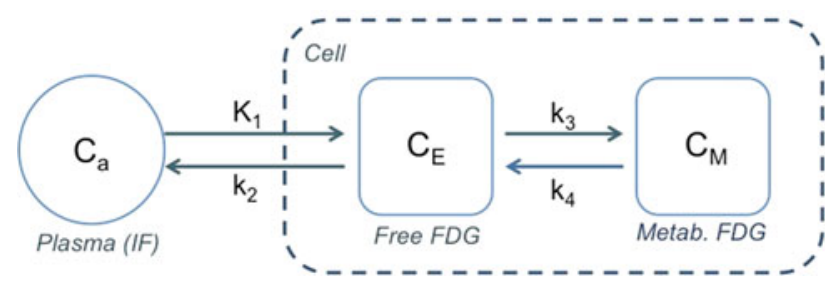

Fig. 1 Compartmental model describing ${ }^{18}$ F-FDG behavior in brain tissue model has also been used. The first tissue compartment, $C_{\mathrm{E}}$, is for free ${ }^{18} \mathrm{~F}-\mathrm{FDG}$, an extravascular pool of tracer exchanging on the one side bidirectionally across the blood-brain barrier with ${ }^{18} \mathrm{~F}-\mathrm{FDG}$ in the plasma, on the other side feeding a final compartment, $C_{\mathrm{M}}$. This second tissue compartment, $C_{\mathrm{M}}$, represents the concentration of ${ }^{18} \mathrm{~F}$-FDG that has been metabolized. This description of the tracer behavior reflects the behavior of glucose up to the phosphorylation step.

Indeed, ${ }^{18} \mathrm{~F}-\mathrm{FDG}$ and glucose in plasma share a common carrier at the blood-brain barrier for transport from plasma into the brain. Once in brain tissue, they compete both for the carrier for transport back from brain to plasma and for the enzyme hexokinase, which phosphorylates ${ }^{18}$ F-FDG to ${ }^{18}$ F-FDG-6-phosphate $\left({ }^{18}\right.$ F-FDG-6-P) in the same manner as it phosphorylates glucose to glucose-6-phosphate. However, because of the substitution in the second carbon position, ${ }^{18} \mathrm{~F}-\mathrm{FDG}-6-\mathrm{P}$ is not a substrate for the next reaction step in the glycolytic pathway, and cannot be metabolized further as glucose-6-phosphate. Furthermore, ${ }^{18}$ F-FDG-6-P is not a significant substrate for glycogen synthesis or for the pentose shunt, and is therefore trapped and unable to leave the cell, except through slow hydrolysis back to free ${ }^{18} \mathrm{~F}-\mathrm{FDG}$, which can then be transported to plasma or be rephosphorylated. This possibility of hydrolysis of ${ }^{18}$ F-FDG-6-P by glucose-6-phosphatase can be incorporated into the model [9]; however, the activity of this enzyme has been demonstrated to be very low in the mammalian brain [7].

The differential equations describing the model with the four rate constants $K_{1}, k_{2}, k_{3}$, and $k_{4}$ (Table 1) are the following:

$\frac{\mathrm{d} C_{\mathrm{E}}}{\mathrm{d} t}=K_{1} C_{\mathrm{a}}-\left(k_{2}+k_{3}\right) C_{\mathrm{E}}+k_{4} C_{\mathrm{M}}$

$\frac{\mathrm{d} C_{\mathrm{M}}}{\mathrm{d} t}=k_{3} C_{\mathrm{E}}-k_{4} C_{\mathrm{M}}$.

Under the assumption that $k_{4}$ is negligibly small [7], regional CMRGlc can be calculated by:

$\mathrm{CMRGlc}=\frac{C_{\mathrm{p}}}{\mathrm{LC}} \frac{K_{1} k_{3}}{\left(k_{2}+k_{3}\right)}$

where $C_{\mathrm{p}}$ is the plasma concentration of glucose, and LC is the lumped constant that accounts for differences in the transport and phosphorylation of ${ }^{18} \mathrm{~F}-\mathrm{FDG}$ and glucose.

$\frac{K_{1} k_{3}}{\left(k_{2}+k_{3}\right)}$ is also defined as $K_{i}$ (units are $\operatorname{mlmin}^{-1} \mathrm{~g}^{-1}$ ), the steady-state influx constant [10], and the equation to calculate CMRGlc becomes:

$\mathrm{CMRGlc}=\frac{C_{\mathrm{p}}}{\mathrm{LC}} K_{i}$.

The kinetic model is based on several key assumptions, including the following: 
- The tracer $\left({ }^{18} \mathrm{~F}-\mathrm{FDG}\right)$ behaves similarly to the substance under investigation (glucose).

- Brain regions are homogeneous with respect to both blood flow and transport rates between plasma and tissue, and phosphorylation rates of glucose and ${ }^{18} \mathrm{~F}$ FDG.

- Glucose metabolism is in a steady state during measurement; i.e., the metabolic rate of glucose, the plasma glucose concentration, and the concentrations of all the substrates and intermediates of the glycolytic pathway are constant throughout the time of study.

- ${ }^{18} \mathrm{~F}-\mathrm{FDG}$ and ${ }^{18} \mathrm{~F}-\mathrm{FDG}-6-\mathrm{P}$ are present in trace amounts; i.e., concentrations of ${ }^{18} \mathrm{~F}-\mathrm{FDG}$ and ${ }^{18} \mathrm{~F}$ FDG-6-P are negligible as compared to their counterparts (glucose and glucose-6-P) and, thus, do not affect the steady-state environment of glucose metabolism.

- The arterial plasma concentrations of glucose and ${ }^{18} \mathrm{~F}$ FDG are approximately equal to their capillary plasma concentrations.

The first four assumptions are fundamental for the application of the model; the last assumption has been added to reduce the complexity of the model, and its validity has been discussed by Sokoloff et al. [7] and Phelps et al. [9].

\section{Quantification methods}

Multiparametric method

The rate constants are mathematically related to the time course of the total activity of ${ }^{18} \mathrm{~F}-\mathrm{FDG}$ in the tissue and the time course of ${ }^{18} \mathrm{~F}$-FDG concentration in arterial plasma. Thus, by using the standard two tissue compartment model and by determining the tissue and arterial plasma concentrations of ${ }^{18} \mathrm{~F}-\mathrm{FDG}$ from arterial sampling and/or dynamic PET data following the intravenous administration of a bolus of ${ }^{18} \mathrm{~F}$-FDG, it is possible to determine the values of $K_{1}, k_{2}, k_{3}, k_{4}$, and thus the value of the steady-state influx constant, $K_{\mathrm{i}}$, by a least-squares non-linear fitting procedure.

The advantage of this technique is that it allows separate calculation of the various rate constants. Its usual disadvantages are its complexity of implementation and longer scan duration.

Given the differential equations describing the ${ }^{18} \mathrm{~F}$ FDG model and considering that the total concentration of ${ }^{18} \mathrm{~F}$-FDG in the tissue is $C_{i}=C_{\mathrm{E}}+C_{\mathrm{M}}$, with scan times $<60 \mathrm{~min}, k_{4}$ is negligibly small, and the solution becomes:

$C_{i}(t)=\frac{K_{1}}{k_{2}+k_{3}}\left(k_{3}+k_{2} e^{-\left(k_{2}+k_{3}\right) t}\right) \otimes C_{\mathrm{a}}(t)$ where $\otimes$ indicates the convolution $f(t) \otimes g(t)=\int_{0}^{t} f(s)$ $g(t-s) \mathrm{d} s$.

Then the kinetic constants $K_{1}, k_{2}$, and $k_{3}$ are estimated by fitting the measured values of $C_{\mathrm{a}}$ and $C_{i}$ in the equation using a non-linear least-squares method. The CMRGlc is finally computed using the Eq. (3) already described: CMRGlc $=\frac{C_{\mathrm{p}}}{\mathrm{LC}} \frac{K_{1} k_{3}}{\left(k_{2}+k_{3}\right)}$.

Sokoloff's autoradiographic method

The first and most commonly used method to estimate CMRGlc using ${ }^{18}$ F-FDG PET is the so-called in vivo autoradiographic approach, it is an adaptation of the 2-deoxy-D-[14C]glucose approach originally developed by Sokoloff et al. [7]. This method was developed for rat brain tissue, and subsequently modified for human studies using PET and ${ }^{18}$ F-FDG by Phelps et al. [9] and Reivich et al. [11]. Sokoloff's autoradiographic method allows a reasonably accurate determination of CMRGlc from singlescan static PET data and a plasma IF.

It is possible to quantify CMRGlc based on single-timepoint PET measurement (performed on tissue sampled at about 45 min after the intravenous tracer administration), given that analysis of the ${ }^{18} \mathrm{~F}$-FDG model shows that the total accumulation of radioactivity in the tissue is predominantly sensitive to the combined forward rate constant and relatively insensitive to assumptions about the exchange of the free pool with plasma.

The measurement of the arterial plasma IF is still needed, so arterial blood sampling is usually performed from the time of injection to the end of the scan to determine the integrated ${ }^{18} \mathrm{~F}$-FDG supply to the tissue.

Finally, by measuring the regional cerebral ${ }^{18} \mathrm{~F}-\mathrm{FDG}$ uptake by means of PET and the time course of arterial plasma ${ }^{18} \mathrm{~F}$-FDG concentration, and knowing the values of the kinetic constants $K_{1}, k_{2}, k_{3}$ (and $k_{4}$, if phosphatase activity is included in the model), and of LC, it is possible to calculate the CMRGlc in the various regions of the brain.

In Sokoloff's method, the $K_{i}$ value in a tissue region or voxel is obtained from the following expression using mean population values for $K_{1}, k_{2}$ and $k_{3}$ and a single cerebral PET scan:

$K_{i}=\frac{C_{i}(T)-K_{1} e^{-\left(k_{2}+k_{3}\right) T} \int_{0}^{T} C_{\mathrm{a}}(t) e^{\left(k_{2}+k_{3}\right) t} \mathrm{~d} t}{\int_{0}^{T} C_{\mathrm{a}}(t) \mathrm{d} t-e^{-\left(k_{2}+k_{3}\right) T} \int_{0}^{T} C_{\mathrm{a}}(t) e^{\left(k_{2}+k_{3}\right) t} \mathrm{~d} t}$

where $C_{\mathrm{a}}(t)$ is the IF, $C_{i}(T)$ is the ${ }^{18} \mathrm{~F}$-FDG uptake in the tissue measured by PET, and $T$ is defined as the mid-time point of the PET scan. This equation is strictly valid for irreversible FDG trapping $\left(k_{4}=0\right)$, while the general equation for $k_{4} \neq 0$ can be found in Phelps et al. [9]. 
Table 1 Kinetic constants of the 18F-FDG compartmental model

\begin{tabular}{ll}
\hline Constant & Description \\
\hline$K_{1}\left(\mathrm{ml} \mathrm{min} \mathrm{m}^{-1}\right)$ & Rate constant of forward transmembranous transport (across blood-brain barrier and cell membrane) \\
$k_{2}\left(\mathrm{~min}^{-1}\right)$ & Rate constant of reversed transmembranous transport \\
$k_{3}\left(\min ^{-1}\right)$ & Rate constant of intra-cytoplasmic 18F-FDG phosphorylation \\
$k_{4}\left(\min ^{-1}\right)$ & Rate constant of intra-cytoplasmic 18F-FDG dephosphorylation \\
\hline
\end{tabular}

Table 2 Literature gray matter mean rate constants

\begin{tabular}{|c|c|c|c|c|c|c|}
\hline & \multicolumn{4}{|c|}{ Irreversible models } & \multicolumn{2}{|c|}{$\begin{array}{l}\text { Reversible } \\
\text { models }\end{array}$} \\
\hline & REIV3 & ROU & HEI & LUC & REIV4 & PHE \\
\hline $\begin{array}{l}K_{1} \\
\quad\left(\mathrm{ml} \mathrm{min} \min ^{-1} \mathrm{~g}^{-1}\right)\end{array}$ & 0.105 & 0.102 & 0.088 & 0.101 & 0.095 & 0.102 \\
\hline$k_{2}\left(\min ^{-1}\right)$ & 0.148 & 0.115 & 0.139 & 0.071 & 0.125 & 0.130 \\
\hline$k_{3}\left(\min ^{-1}\right)$ & 0.074 & 0.037 & 0.066 & 0.042 & 0.069 & 0.062 \\
\hline$k_{4}\left(\min ^{-1}\right)$ & 0 & 0 & 0 & 0 & 0.0055 & 0.0068 \\
\hline
\end{tabular}

Table 2 gives some examples of gray matter mean rate constants derived from the literature, considering both irreversible and reversible models (REIV3 and REIV4 [12], ROU [13], HEI [14], LUC [15], PHE [9]).

The Sokoloff autoradiographic method, with or without the inclusion of $k_{4}$, has the advantages of short PET scan times (with modern PET scanners about 10-20 min) and low variance of CMRGlc values at voxel level. However, the main disadvantages are that individual kinetic rate constants cannot be determined using this technique and the use of predetermined kinetic constants can lead to biases in CMRGlc calculation; indeed, it has been proved that if the standard values differ greatly from those of the subject under examination, as in the case of severely affected subjects, then the $K_{i}$ estimates can be strongly biased [16-18]. In order to reduce this problem, several authors proposed alternative equations for single-scan estimation of CMRGlc; the most frequently used has been described by Hutchins et al. [19].

Gjedde-Patlak graphical method

In general, graphical analyses are a further simplification of general linear methods: they convert the model equations into one equation, which is evaluated at time points corresponding to the scanning times, thus providing fewer parameters, namely, a slope and intercept.

The Gjedde-Patlak graphical method can be used to estimate the combination of the kinetic rate constants, which is the steady-state influx constant $K_{i}$ under the hypothesis that in the tissue there is a compartment in which the tracer is irreversibly entrapped [20]. This compartment can accommodate multiple reversible compartments prior to irreversible trapping, thus reducing the demands on tissue homogeneity.

Knowing $K_{i}$, CMRGlc can be calculated in combination with the plasma glucose concentration and the LC (see equation above).

The requirements are a dynamic PET scan to obtain a series of measured tissue ${ }^{18}$ F-FDG activity over time and an arterial plasma IF.

Assuming unidirectional uptake of ${ }^{18}$ F-FDG $\left(k_{4}=0\right)$, a graph of the ratio of the ${ }^{18} \mathrm{~F}$ tissue concentration to the plasma ${ }^{18} \mathrm{~F}$ concentration versus the ratio of the plasma ${ }^{18} \mathrm{~F}$ concentration time integral to the plasma ${ }^{18} \mathrm{~F}$ concentration yields a curve that eventually approaches a straight line with the slope equal to $K_{i}$. At sufficiently late times $\left(t^{*}\right)$ when the reversible compartments are in equilibrium with plasma concentration $C_{\mathrm{a}}, K_{i}$ is obtained through the linear fit of the following expression:

$\frac{C_{i}(t)}{C_{\mathrm{a}}(t)}=K_{i} \frac{\int_{0}^{t} C_{\mathrm{a}}(\tau) \mathrm{d} \tau}{C_{\mathrm{a}}(t)}+\left(V_{0}+V_{\mathrm{p}}\right) \quad$ for $t \geq t *$

where $C_{i}(t)$ is the uptake curve in the tissue measured through a dynamic PET acquisition. The intercept is the sum of $V_{0}=K_{1} k_{2} /\left(k_{2}+k_{3}\right)^{2}$ and the vascular fraction in the tissue, $V_{\mathrm{p}}$. It is worth noting that by using the Patlak approach, $V_{\mathrm{p}}$ is not being neglected as it is when fitting rate constants or when using a static operational equation on the assumption that the vascular space in tissue is small as compared to the extravascular space [9]; instead with the Patlak plot approach, $V_{\mathrm{p}}$ is specifically included in the intercept value. Since this equation has been derived by the author in the hypothesis that there is no significant loss of metabolic product, in this case $k_{4}$ must be zero to guarantee the linearity of the plot. A modification of this method has been proposed to accommodate for $k_{4} \neq 0$, although it is not widely used and lacks the simplicity of the original method [10].

This approach has several advantages as compared to the multiparametric method; for example, it has superior variance properties, giving more robust CMRGlc measures, and it can easily generate parametric images. The main disadvantages are that individual values of kinetic rate constants cannot be determined, and that it has an inherent systemic bias due to the fact that the plot only approximates but is not a straight line, even under ideal circumstances with $k_{4}=0$. 
As compared to the autoradiographic method, the main advantage is that the Patlak method gives more personalized estimates of CMRGlc, even in very diseased states; however, a disadvantage is that parametric images generated with the Patlak method are more affected by noise than Sokoloff ones. Another disadvantage is that it still requires dynamic PET scanning, which does not necessarily need to start from the time of injection, but would be longer compared with single-scan procedures, in order to obtain a sufficient number of measurements of ${ }^{18} \mathrm{~F}$-FDG tissue concentration to fit a reliable straight line from which to derive the $K_{i}$.

\section{Lumped constant}

${ }^{18} \mathrm{~F}-\mathrm{FDG}$ and glucose differ significantly in their membrane transport, phosphorylation and distribution volumes in brain tissue [9]. The parameter that accounts for such differences is called the lumped constant, and it is based on the biochemical principles of competitive substrate kinetics. The value of the LC is used to convert the metabolic rate of ${ }^{18} \mathrm{~F}$-FDG to the metabolic rate of glucose; it is clear that its value is critical in quantitative calculation of CMRGlc.

The LC is commonly assumed to be uniform over the whole brain and under normal conditions in all subjects. However, there are several conditions in which LC may be altered, such as in tumors, the developing brain and in conditions of limited supply (hypoglycemia and ischemia) $[21,22]$.

The LC is a function of $C_{\mathrm{p}}$ and of the rate constants; the formula of the LC defined by Sokoloff et al. [7] is:

$\mathrm{LC}=\frac{\lambda \cdot K_{\mathrm{m}} \cdot V_{\max }^{*}}{\phi \cdot V_{\max } \cdot K_{\mathrm{m}}^{*}}$

where $\lambda$ is the ratio of the distribution volume of ${ }^{18} \mathrm{~F}$-FDG to that of glucose, $\Phi$ is the fraction of glucose that continues down the metabolic pathway after being phosphorylated, $K_{\mathrm{m}}$ is the Michaelis-Menten constant for phosphorylation of glucose (* indicates for ${ }^{18} \mathrm{~F}-\mathrm{FDG}$ ), and $V_{\max }$ is the maximum velocity for phosphorylation of glucose $\left(*\right.$ indicates for $\left.{ }^{18} \mathrm{~F}-\mathrm{FDG}\right)$.

The LC value for ${ }^{18} \mathrm{~F}$-FDG in cerebral tissue in humans has been extensively studied using several techniques and yielding different values (Table 3); the variability of LC values reported in the literature could be attributed to the different methodologies used. For brain studies, the recommended LC values are 0.65 if irreversible uptake is assumed $\left(k_{4}=0\right)$, and 0.81 , if reversible uptake is assumed $\left(k_{4} \neq 0\right)[23]$.
Table 3 LC values reported in the literature

\begin{tabular}{ll}
\hline Study & LC \\
\hline Reivich et al. [11] & 0.52 \\
Phelps et al. [9] & 0.42 \\
Brooks et al. [34] & 0.50 \\
Gjedde et al. [35] & 0.42 \\
Hasselbalch et al. [36] & 0.48 \\
Hasselbalch et al. [36] & 0.81 \\
Lammertsma et al. [37] & 0.75 \\
Graham et al. [38] & 0.89 \\
\hline
\end{tabular}

\section{Input function}

All the CMRGlc calculation methods described above require assessment of the IF, which is an estimate of the time course of the delivery of ${ }^{18}$ F-FDG to the brain. The literature describes different approaches for obtaining the IF.

The gold-standard method is to catheterize an artery of the subject and take a series of blood samples following injection of the tracer. The blood samples must be obtained over the full-time course of the scan and with a frequency and over a length of time adequate to define the level of activity and shape of the IF.

It is not, however, always possible or desirable to sample arterial blood and there are a number of complications that commonly occur when obtaining the IF with this procedure, thereby making it more problematic in clinical settings or in large-scale studies.

First of all, arterial blood sampling involves the insertion of arterial lines, which is quite invasive, uncomfortable for the patient, and involves potential risks, albeit fairly infrequent [24]. Furthermore, extra personnel are needed for the sampling procedure and processing of arterial blood, which increases the risks associated with blood and radiation exposure to support personnel and also necessitates additional laboratory procedures for measuring the blood samples and calibrating the equipment.

For these reasons, over recent decades, other methods have been developed to avoid arterial puncture in the measurement of the IF, even though this remains the goldstandard method.

One of the alternative methods is catheterization of a distal vein and sampling of arterialized venous blood. The rationale for this procedure is the fact that, when the temperature of the hand is raised, artero-venous shunts open and the blood in the surface veins is effectively arterialized. The main disadvantages of this procedure are: (1) venous access in the hand to obtain arterialized venous blood can be problematic; (2) it is not always possible to 
maintain consistent sampling, timing, and volume across experiments; (3) the procedure is known to produce much blunted functions [25].

Alternatives to serial arterial or venous sampling are modeling of a population-based IF [26, 27] or extraction of an image-derived IF (IDIF) [28, 29]. Population-based curves reduce the need for blood sampling and can be based on averaging of normalized blood curves or on fitting to a proposed equation [26].

The IDIF procedure uses the PET system to measure the IF directly from the images by measuring the amount of activity in the left ventricle, aorta, carotid arteries or other large arteries as a function of time. Nowadays, PET systems are capable of accumulating images rapidly enough to satisfy the temporal sampling requirements of the particular IFs.

The IDIFs have been extensively studied and found to satisfy the criteria for obtaining a reasonably good IF.

The use of an IDIF is attractive for several reasons. First, it eliminates a common source of error-the need for precise cross-calibration between a dose calibrator and the PET scanner. Second, by providing the equivalent of an arterial IF without blood sampling during the acquisition, IDIF makes the study simpler, reduces patient risk and discomfort, involves fewer manipulations, reduces personnel requirements, and lowers the risk of occupational hazards related to blood handling. Third, serial blood sampling can also be a deterrent to subject participation when venous or arterial access is problematic, i.e., in the elderly.

The use of carotid arteries to obtain the IDIF for brain studies has already been explored by several groups [24, $30,31]$. One of its advantages is the presence of carotid arteries in the brain volume acquired with the dynamic PET, which means that both the arterial and the brain uptake can be measured from their very start. However, the inner diameter of the carotid arteries is similar to the PET spatial resolution; therefore, IDIF measured in this way will suffer from the partial volume effect in the initial phase and spill-in from adjacent brain tissue later on, making it necessary to apply laborious correction methods [24, 31, 32].

The aorta would offer advantages in this respect due to its larger inner diameter and to the fact that much of its path is far from tissues with high ${ }^{18} \mathrm{~F}$-FDG uptake, meaning that spill-in corrections are often negligible [33]. However, because of the distance from the brain, the cerebral uptake cannot be monitored from its start; as a result, this approach is suitable only in those cases in which determination of the $K_{i}$ is sufficient, and the individual kinetic constants are not needed.

These IDIF methods still suffer from errors ultimately leading to less reliable CMRGlc measurements [32]. Blood sample-free methods provide rather biased estimation of CMRGlc, with variations $> \pm 10 \%$ as compared to the CMRGlc values obtained with the gold-standard IF method (with arterial sampling). CMRGlc estimates consistently improve if the IDIF is calibrated with venous blood samples, leading to reliable CMRGlc calculations with variations less than $5 \%$ as compared to the gold-standard arterial sampling IF.

\section{Conclusions}

${ }^{18}$ F-FDG PET is a powerful technique for studying in vivo the physiology and biochemistry of the human brain. It can be used to perform quantitative measurements of CMRGlc with reasonable levels of accuracy.

Ever since its introduction, PET has been a popular quantitative tool; however, while the initial quantitative approaches with brain ${ }^{18} \mathrm{~F}$-FDG PET were already reliable, they were also technically demanding and, thus, considered impractical for routine use in clinical settings.

Over recent decades, quantitative brain ${ }^{18} \mathrm{~F}$-FDG PET has undergone constant refinements to develop easy yet accurate CMRGlc quantification methods, able to complement visual image interpretation and minimize interobserver variability. Its main applications have been linked to the use of modern PET scanners with high spatial and temporal resolution, and the development of simplified methods to derive the IF directly from PET images. Further refinements are still needed to develop simple yet accurate procedures that can be applied not only in research and clinical trials, but also in clinical settings.

Conflict of interest V. Berti, E. Vanzi, C. Polito, A. Pupi declare that they have no conflict of interest.

Human and Animal Studies This article does not contain any studies with human or animal subjects performed by any of the authors.

\section{References}

1. Mosconi L, Tsui WH, Herholz K et al (2008) Multicenter standardized 18F-FDG PET diagnosis of mild cognitive impairment, Alzheimer's disease, and other dementias. J Nucl Med 49:390398. doi:10.2967/jnumed.107.045385

2. Bohnen NI, Djang DS, Herholz K et al (2012) Effectiveness and safety of 18F-FDG PET in the evaluation of dementia: a review of the recent literature. J Nucl Med 53:59-71. doi:10.2967/ jnumed.111.096578

3. Silverman DH (2004) Brain 18F-FDG PET in the diagnosis of neurodegenerative dementias: comparison with perfusion SPECT and with clinical evaluations lacking nuclear imaging. J Nucl Med 45:594-607

4. Mosconi L, Mistur R, Switalski R et al (2009) FDG-PET changes in brain glucose metabolism from normal cognition to 
pathologically verified Alzheimer's disease. Eur J Nucl Med Mol Imaging 36:811-822. doi:10.1007/s00259-008-1039-Z

5. Schmidt R, Ropele S, Pendl B et al (2008) Longitudinal multimodal imaging in mild to moderate Alzheimer disease: a pilot study with memantine. J Neurol Neurosurg Psychiatry 79:13121317

6. Potkin SG, Anand R, Fleming K et al (2001) Brain metabolic and clinical effects of rivastigmine in Alzheimer's disease. Int $\mathrm{J}$ Neuropsychopharmacol 4:223-230. doi:10.1017/S146114570100 2528

7. Sokoloff L, Reivich M, Kennedy C et al (1977) The [14C]deoxyglucose method for the measurement of local cerebral glucose utilization: theory, procedure, and normal values in the conscious and anesthetized albino rat. J Neurochem 28:897-916

8. Freygang WH Jr, Sokoloff L (1958) Quantitative measurement of regional circulation in the central nervous system by the use of radioactive inert gas. Adv Biol Med Phys 6:263-279

9. Phelps ME, Huang SC, Hoffman EJ et al (1979) Tomographic measurement of local cerebral glucose metabolic rate in humans with (F-18)2-fluoro-2-deoxy-D-glucose: validation of method. Ann Neurol 6:371-388

10. Patlak CS, Blasberg RG (1985) Graphical evaluation of blood-tobrain transfer constants from multiple-time uptake data. Generalizations. J Cereb Blood Flow Metab 5:584-590

11. Reivich M, Kuhl D, Wolf A et al (1979) The [18F]fluorodeoxyglucose method for the measurement of local cerebral glucose utilization in man. Circ Res 44:127-137

12. Reivich M, Alavi A, Wolf A et al (1985) Glucose metabolic rate kinetic model parameter determination in humans: the lumped constants and rate constants for $[18 \mathrm{~F}]$ fluorodeoxyglucose and [11C]deoxyglucose. J Cereb Blood Flow Metab 5:179-192

13. Rougemont D, Baron JC, Collard P et al (1984) Local cerebral glucose utilisation in treated and untreated patients with Parkinson's disease. J Neurol Neurosurg Psychiatry 47:824-830

14. Heiss WD, Pawlik G, Herholz K et al (1984) Regional kinetic constants and cerebral metabolic rate for glucose in normal human volunteers determined by dynamic positron emission tomography of [18F]-2-Fluoro-2-deoxy-D-glucose. J Cereb Blood Flow Metab 4:212-223

15. Lucignani G, Schmidt KC, Moresco RM et al (1993) Measurement of regional cerebral glucose utilization with fluorine-18FDG and PET in heterogeneous tissues: theoretical considerations and practical procedure. J Nucl Med 34:360-369

16. Huang SC, Phelps ME, Hoffman EJ et al (1980) Noninvasive determination of local cerebral metabolic rate of glucose in man. Am J Physiol 238:E69-E82

17. Huang S-C, Phelps ME, Hoffman EJ, Kuhl DE (1981) Error sensitivity of fluorodeoxyglucose method for measurement of cerebral metabolic rate of glucose. J Cereb Blood Flow Metab $1: 391-401$

18. Wienhard K, Pawlik G, Herholz K et al (1985) Estimation of local cerebral glucose utilization by positron emission tomography of [18F]2-fluoro-2-deoxy-D-glucose: a critical appraisal of optimization procedures. J Cereb Blood Flow Metab 5:115-125. doi:10.1038/jcbfm.1985.15

19. Hutchins GD, Holden JE, Koeppe RA et al (1984) Alternative approach to single-scan estimation of cerebral glucose metabolic rate using glucose analogs, with particular application to ischemia. J Cereb Blood Flow Metab 4:35-40. doi:10.1038/jcbfm. 1984.5

20. Patlak CS, Blasberg RG, Fenstermacher JD (1983) Graphical evaluation of blood-to-brain transfer constants from multipletime uptake data. J Cereb Blood Flow Metab 3:1-7

21. Crane PD, Pardridge WM, Braun LD et al (1981) The interaction of transport and metabolism on brain glucose utilization: a reevaluation of the lumped constant. J Neurochem 36:1601-1604

22. Hawkins RA, Phelps ME, Huang SC, Kuhl DE (1981) Effect of ischemia on quantification of local cerebral glucose metabolic rate in man. J Cereb Blood Flow Metab 1:37-51. doi:10.1038/ jcbfm.1981.5

23. Wu H, Bergsneider M, Glenn TC et al (2003) Measurement of the global lumped constant for 2-Deoxy-2-[18F]fluoro-D-glucose in normal human brain using [15O]water and 2-Deoxy-2-[18F]fluoro-D-glucose positron emission tomography imaging. A method with validation based on multiple methodologies. Mol Imaging Biol 5:32-41. doi:10.1016/S1536-1632(02)00122-1

24. Chen K, Bandy D, Reiman E et al (1998) Noninvasive quantification of the cerebral metabolic rate for glucose using positron emission tomography, 18F-fluoro-2-deoxyglucose, the Patlak method, and an image-derived input function. J Cereb Blood Flow Metab 18:716-723. doi:10.1097/00004647-19980700000002

25. van der Weerdt AP, Klein LJ, Visser CA et al (2002) Use of arterialised venous instead of arterial blood for measurement of myocardial glucose metabolism during euglycaemic-hyperinsulinaemic clamping. Eur $\mathrm{J}$ Nucl Med Mol Imaging 29:663-669. doi:10.1007/s00259-002-0772-y

26. Eberl S, Anayat AR, Fulton RR et al (1997) Evaluation of two population-based input functions for quantitative neurological FDG PET studies. Eur J Nucl Med 24:299-304

27. Shiozaki T, Sadato N, Senda M et al (2000) Noninvasive estimation of FDG input function for quantification of cerebral metabolic rate of glucose: optimization and multicenter evaluation. J Nucl Med 41:1612-1618

28. Ohtake T, Kosaka N, Watanabe T et al (1991) Noninvasive method to obtain input function for measuring tissue glucose utilization of thoracic and abdominal organs. J Nucl Med 32:1432-1438

29. de Geus-Oei L-F, Visser EP, Krabbe PFM et al (2006) Comparison of image-derived and arterial input functions for estimating the rate of glucose metabolism in therapy-monitoring 18F-FDG PET studies. J Nucl Med 47:945-949

30. Zanotti-Fregonara P, Maroy R, Comtat C et al (2009) Comparison of 3 methods of automated internal carotid segmentation in human brain PET studies: application to the estimation of arterial input function. J Nucl Med 50:461-467. doi:10.2967/jnumed.108. 059642

31. Croteau E, Lavallée E, Labbe SM et al (2010) Image-derived input function in dynamic human PET/CT: methodology and validation with $11 \mathrm{C}$-acetate and $18 \mathrm{~F}$-fluorothioheptadecanoic acid in muscle and $18 \mathrm{~F}$-fluorodeoxyglucose in brain. Eur J Nucl Med Mol Imaging 37:1539-1550. doi:10.1007/s00259-010$1443-\mathrm{Z}$

32. Zanotti-Fregonara P, el Fadaili M, Maroy R et al (2009) Comparison of eight methods for the estimation of the image-derived input function in dynamic [18F]-FDG PET human brain studies. J Cereb Blood Flow Metab 29:1825-1835. doi:10.1038/jcbfm. 2009.93

33. Dhawan V, Takikawa S, Robeson W et al (1994) Quantitative brain FDG/PET studies using dynamic aortic imaging. Phys Med Biol 39:1475-1487

34. Brooks RA, Hatazawa J, Di Chiro G et al (1987) Human cerebral glucose metabolism determined by positron emission tomography: a revisit. J Cereb Blood Flow Metab 7:427-432

35. Gjedde A, Wienhard K, Heiss WD et al (1985) Comparative regional analysis of 2-fluorodeoxyglucose and methylglucose uptake in brain of four stroke patients. With special reference to the regional estimation of the lumped constant. J Cereb Blood Flow Metab 5:163-178 
36. Hasselbalch SG, Holm S, Pedersen HS et al (2001) F-fluorodeoxyglucose lumped constant determined in human brain from extraction fractions of $18 \mathrm{~F}$-fluorodeoxyglucose and glucose. J Cereb Blood Flow Metab 21:995-1002

37. Lammertsma AA, Brooks DJ, Frackowiak RS et al (1987) Measurement of glucose utilisation with [18F]2-fluoro-2-deoxy-
D-glucose: a comparison of different analytical methods. J Cereb Blood Flow Metab 7:161-172

38. Graham MM, Muzi M, Spence AM et al (2002) The FDG lumped constant in normal human brain. Journal of Nuclear Medicine 43:1157-1168 